\title{
Respostas fisiológicas da cana-de-açúcar com suplementação potássica em dois regimes hídricos
}

\author{
Physiological responses of sugarcane with potassium supplementation in two water regimes \\ Respuestas fisiológicas de la caña de azúcar con suplementación de potasio en dos regímenes \\ hídricos
}

Pedro Henrique Ribeiro ORCID: https://orcid.org/0000-0001-5867-3310

Key Account Manager, Brasil

E-mail: pedro.ribeiro@valmont.com

Glauber José de Castro Gava

ORCID: https://orcid.org/0000-0002-3194-5432

Polo Regional Centro Oeste/APTA, Brasil

E-mail: ggava@apta.sp.gov.br

Adolfo Bergamo Arlanch

ORCID: https://orcid.org/0000-0001-8171-8265

Universidade Estadual do Norte do Paraná, Brasil

E-mail: adolfoarlanch@gmail.com

Marconi Batista Teixeira

ORCID: https://orcid.org/0000-0002-0152-256X

Instituto Federal de Educação, Ciência e Tecnologia Goiano, Brasil

E-mail: marconi.teixeira@ifgoiano.edu.br

Evaldo Alves dos Santos

ORCID: https://orcid.org/0000-0001-5610-4288

Instituto Federal de Educação, Ciência e Tecnologia Goiano, Brasil

E-mail: evaldo0.santos@gmail.com

Fernando Rodrigues Cabral Filho

ORCID: https://orcid.org/0000-0002-5090-5946 Instituto Federal de Educação, Ciência e Tecnologia Goiano, Brasil

E-mail: fernandorcfilho@hotmail.com

João Alberto Lelis Neto

ORCID: https://orcid.org/0000-0001-6599-5924 Zeus Agrotech, Brasil

E-mail: joao_lelis@yahoo.com.br

Fernando Nobre Cunha

ORCID: https://orcid.org/0000-0001-8489-7625 Instituto Federal de Educação, Ciência e Tecnologia Goiano, Brasil

E-mail: fernandonobrecunha@ hotmail.com

Daniely Karen Matias Alves

ORCID: https://orcid.org/0000-0001-7427-7545

Dantas Soluções Ambientais e Industriais, Brasil

E-mail: daniely_karen@hotmail.com

Alefe Viana Souza Bastos

ORCID: https://orcid.org/0000-0002-0349-2421

Terram Soluções Integradas, Brasil

E-mail: alefe_viana@hotmail.com

Edson Cabral da Silva

ORCID: https://orcid.org/0000-0002-1813-490X Instituto Federal de Educação, Ciência e Tecnologia Goiano, Brasil

E-mail: edsoncabralsilva@gmail.com

Vitor Marques Vidal

ORCID: https://orcid.org/0000-0001-5179-6684 Instituto Federal de Educação, Ciência e Tecnologia Goiano, Brasil

E-mail: vmarquesvidal@gmail.com

Wilker Alves Morais

ORCID: https://orcid.org/0000-0003-2336-6518 Instituto Federal de Educação, Ciência e Tecnologia Goiano, Brasil E-mail: wilker.alves.morais@gmail.com 


\begin{abstract}
Resumo
A cana-de-açúcar é uma das principais culturas exploradas no Brasil, seja em condições de sequeiro ou irrigada. A água e o nutriente potássio são insumos que podem interferir na físiologia dessa cultura e, quando fornecido em fertirrigação, permite uma maior eficiência do uso da água e de fertilizantes. O potássio é o nutriente mais exigido pela cana-de-açúcar e a sua eficiência é melhorada quando se utiliza a fertirrigação. Assim, objetivou-se avaliar as respostas fisiológicas da cana-de-açúcar durante o terceiro ciclo de cultivo (soqueira) quanto à aplicação de doses de potássio em condições de sequeiro e irrigação. O experimento foi desenvolvido em esquema de parcelas subdivididas (4 x 2), sendo quatro doses de adubação potássica $\left(0,70,140\right.$ e $210 \mathrm{~kg} \mathrm{ha}^{-1}$ de $\mathrm{K}_{2} \mathrm{O}$ ) com e sem irrigação. A condutância estomática foi maior quando as plantas de cana-de-açúcar foram irrigadas em todas as doses de potássio estudadas, exceto para o controle aos 235 e 393 dias após o corte. O índice SPAD não apresentou diferença entre as condições irrigado e sequeiro em todas as doses de adubação potássica e épocas de avaliação. Há diferença no índice de área foliar entre as plantas irrigadas e em condições de sequeiro apenas quando se aplica a dose de $140 \mathrm{~kg}^{-1} \mathrm{de}$ $\mathrm{K}_{2} \mathrm{O}$ aos 340 dias após o corte.
\end{abstract}

Palavras-chave: Saccharum officinarum; Fertirrigação; Potássio; Irrigação.

\begin{abstract}
Sugarcane is one of the main crops explored in Brazil, whether under rainfed or irrigated conditions. Water and the nutrient potassium are inputs that can interfere with the physiology of this crop and, when supplied in fertigation, allows for greater efficiency in the use of water and fertilizers. Potassium is the nutrient most required by sugarcane and its efficiency is improved when fertigation is used. Thus, the objective was to evaluate the physiological responses of sugarcane during the third cropping cycle (stump) regarding the application of potassium doses under rainfed and irrigation conditions. The experiment was carried out in a split-plot scheme (4 $\mathrm{x} 2$ ), with four doses of potassium fertilization $\left(0,70,140\right.$ and $210 \mathrm{~kg} \mathrm{ha}^{-1}$ of $\left.\mathrm{K}_{2} \mathrm{O}\right)$ with and without irrigation. The stomatal conductance was higher when the sugarcane plants were irrigated at all doses of potassium studied, except for the control at 235 and 393 days after cutting. The SPAD index showed no difference between irrigated and dryland conditions in all doses of potassium fertilization and evaluation periods. There is a difference in the leaf area index between irrigated plants and under rainfed conditions only when the dose of $140 \mathrm{~kg} \mathrm{ha}^{-1}$ of $\mathrm{K}_{2} \mathrm{O}$ is applied 340 days after cutting.
\end{abstract}

Keywords: Saccharum officinarum; Fertigation; Potassium; Irrigation.

\title{
Resumen
}

La caña de azúcar es uno de los principales cultivos explorados en Brasil, ya sea de secano o de regadío. El agua y el nutriente potasio son insumos que pueden interferir con la fisiología de este cultivo y, al ser suministrados en fertirrigación, permiten una mayor eficiencia en el uso de agua y fertilizantes. El potasio es el nutriente más requerido por la caña de azúcar y su eficiencia mejora cuando se usa fertirrigación. Así, el objetivo fue evaluar las respuestas fisiológicas de la caña de azúcar durante el tercer ciclo de cultivo (tocón) respecto a la aplicación de dosis de potasio en condiciones de secano y riego. El experimento se realizó en un esquema de parcelas divididas (4 x 2), con cuatro dosis de fertilización potásica $\left(0,70,140\right.$ y $210 \mathrm{~kg} \mathrm{ha}^{-1}$ de $\left.\mathrm{K}_{2} \mathrm{O}\right)$ con y sin riego. La conductancia estomática fue mayor cuando las plantas de caña de azúcar fueron irrigadas con todas las dosis de potasio estudiadas, excepto para el control a los 235 y 393 días después del corte. El índice SPAD no mostró diferencias entre las condiciones de regadío y de secano en todas las dosis de fertilización con potasio y períodos de evaluación. Existe una diferencia en el índice de área foliar entre plantas irrigadas y de secano solo cuando se aplica la dosis de $140 \mathrm{~kg} \mathrm{ha}^{-1}$ de $\mathrm{K}_{2} \mathrm{O} 340$ días después del corte.

Palabras clave: Saccharum officinarum; Fertirrigación; Potasio; Riego.

\section{Introdução}

A cana-de-açúcar (Saccharum officinarum) é uma cultura agrícola explorada, comercialmente, em mais de 79 países e, ocupa uma área de aproximadamente 26,4 milhões de hectares, com destaque para Brasil, Cuba, Índia, México, China, Filipinas, Austrália, África do Sul, Estados Unidos da América (principalmente Havaí) e República Dominicana (Conab, 2016).

Diversos fatores influenciam no rendimento e na maturação da cana-de-açúcar como, por exemplo, a interação edafoclimática, os manejos da irrigação e da fertirrigação, bem como a cultivar explorada (Oliveira, 2011).

Uma vez que, a frequência e a intensidade hídrica interferem na produção das culturas, há uma maior iniciativa com o intuito de melhorar a produtividade de cultivos agrícolas sob condições de seca (Cattivelli et al., 2008). Exceto quando se utilizam a irrigação, a disponibilidade hídrica, a limitação as águas pluviais e subterrâneas e, mesmo com o avanço das técnicas de irrigação, essa prática para a produção de cana-de-açúcar ainda é pouco difundida, embora os benefícios à cultura sejam 
inúmeros (Gava et al., 2011). A irrigação evita o déficit hídrico das culturas quando manejada adequadamente, no entanto, para obtenção de resultados satisfatórios, faz-se necessário associá-la a outras práticas culturais como, por exemplo, a fertirrigação (Dalri \& Cruz, 2008).

Em relação à exigência nutricional, a cana-de-açúcar utiliza-se de 13\% do total de fertilizantes aplicados anualmente no Brasil, equivalente a 2,9 milhões de toneladas. O nitrogênio e, principalmente, o potássio são os nutrientes mais exigidos pela cana-de-açúcar, de modo que para cada $100 \mathrm{t} \mathrm{ha}^{-1}$ de colmos, são exportados cerca de $150 \mathrm{~kg} \mathrm{ha}^{-1}$ de $\mathrm{K}_{2} \mathrm{O}$ (Malavolta, 1982).

A literatura reporta que há interações entre a deficiência hídrica e a absorção de íons K sobre a interceptação de luz e a produtividade primária bruta. A deficiência hídrica, frequentemente está associada a uma perturbação na homeostase de íons de $\mathrm{K}^{+}$intracelular, porque a seca aumenta tanto as exigências de K como diminui a absorção de K (Oddo et al., 2014, Shabala \& Pottosin, 2014). Algumas pesquisas, sugerem que a adubação com K pode aliviar os efeitos adversos do estresse hídrico, desde que o efeito positivo sobre a regulação estomática não seja compensado posteriormente por um maior uso da água (Taulya, 2013).

Levando-se em consideração estes aspectos, objetivou-se avaliar as respostas fisiológicas da cana-de-açúcar durante o segundo ciclo de cultivo (soqueira) quanto à aplicação de doses de potássio em condições de sequeiro e irrigação.

\section{Metodologia}

O experimento foi desenvolvido em campo aberto na Unidade de Pesquisa e Desenvolvimento Hélio de Moraes da

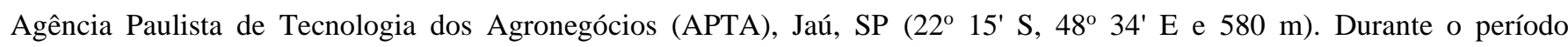
experimental, foram registradas temperatura média anual mínima de $17,53{ }^{\circ} \mathrm{C}$ e a máxima em $29,03{ }^{\circ} \mathrm{C}$, tornando o ambiente favorável e propicio a cultura (Figura 1).

Figura 1. Curvas de temperatura mínima, máxima e média durante a realização do experimento e utilizadas no manejo da cana-soca.

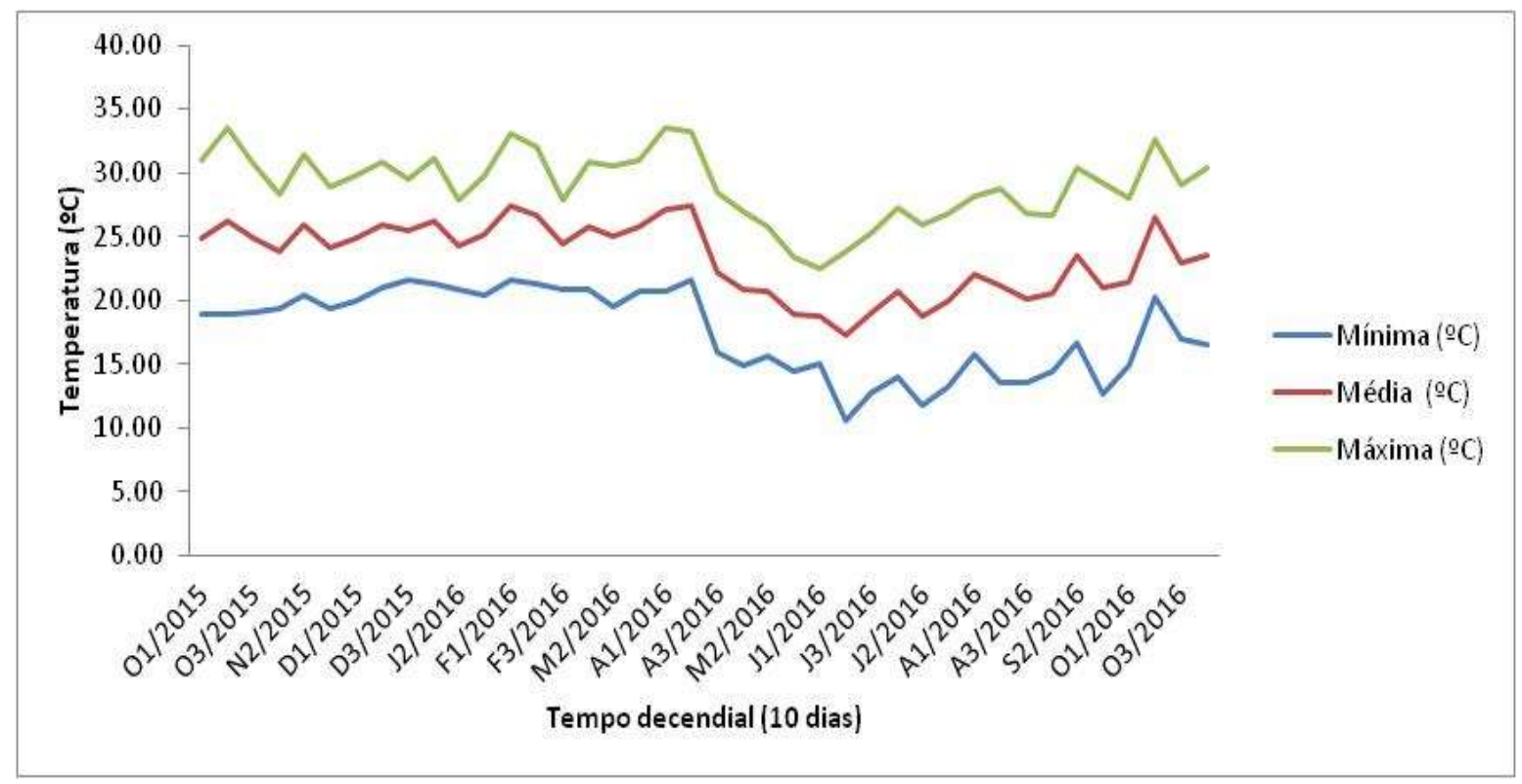

Fonte: Autores. Estação meteorológica da FATEC de Jaú/SP. 
O experimento ocorreu durante 393 dias após o corte (DAC) - segundo ciclo da cana-soca, compreendendo o período de 07 de outubro de 2015 a 03 de novembro de 2016. O solo da área foi classificado como Latossolo Vermelho (Silva et al., 2014) com textura média na camada superior e argilosa nas camadas de 20 a $60 \mathrm{~cm}$ e densidade média de $2,75 \mathrm{~g} \mathrm{~cm}^{-3}$. Amostras de solos nas camadas de 0-20, 20-40 e 40-60 cm foram coletadas para caracterização físico-química (Tabela 1).

Tabela 1. Análise química do solo da área experimental nas diferentes camadas.

\begin{tabular}{ccccccccccccc}
\hline \multirow{2}{*}{ Prof. (cm) } & $\mathbf{p H}$ & $\mathbf{M . O}$ & $\mathbf{P}_{\text {resina }}$ & $\mathbf{S}$ & $\mathbf{A l}^{3+}$ & $\mathbf{H}+\mathbf{A l}$ & $\mathbf{K}$ & $\mathbf{C a}$ & $\mathbf{M g}$ & $\mathbf{S B}$ & $\mathbf{C T C}$ & $\mathbf{V \%}$ \\
\cline { 2 - 11 } & $\mathrm{CaCl}_{2}$ & $\mathrm{~g} \mathrm{dm}^{-3}$ & \multicolumn{2}{c}{$\mathrm{mg} \mathrm{dm}^{-3}$} & & & $\mathrm{mmol}_{\mathrm{c}} \mathrm{dm}^{-3}$ & & & & \\
\hline $0-20$ & $5,5^{*}$ & 15 & 28 & 10 & 0 & 24 & 0,7 & 21 & 13 & 35 & 60 & 59 \\
$20-40$ & 4,2 & 11 & 23 & 50 & 5 & 56 & 0,2 & 11 & 5 & 17 & 73 & 23 \\
$40-60$ & 4,1 & 10 & 14 & 49 & 6 & 63 & 0,2 & 15 & 5 & 20 & 83 & 24 \\
\hline
\end{tabular}

Fonte: Laboratório de Fertilidade do Solo da Faculdade de Ciências Agronômicas da UNESP, Botucatu, SP. Análise realizada segundo metodologia de Raij et al. (2001).

O delineamento experimental foi o de blocos ao acaso em esquema de parcelas subdivididas (4 $\mathrm{x} 2$ ), sendo quatro doses de $\mathrm{K}_{2} \mathrm{O}\left(0,70,140,210 \mathrm{~kg} \mathrm{ha}^{-1}\right)$ e duas condições hídricas (irrigação e sequeiro), com quatro repetições. As parcelas foram formadas de dez linhas de $8 \mathrm{~m}$ de comprimento, sendo utilizados para avaliação apenas os 6 linhas centrais, em que as demais serviram como bordadura, com plantio em linha dupla (ou em "W" com 0,40 m entre linhas), espaçamento de 1,80 m entre as linhas duplas e sistema de irrigação por gotejamento subsuperficial. A parcela experimental totalizou $281,6 \mathrm{~m}^{2}(9 \mathrm{~m}$ largura x $32 \mathrm{~m}$ comprimento) e foi composta de 5 linhas duplas.

Todos os tratamentos receberam a mesma dose de nitrogênio correspondendo a $210 \mathrm{~kg}$ de $\mathrm{N}$ via ureia. A fonte de adubação potássica foi o cloreto de potássio branco. Nos tratamentos irrigados a aplicação de $\mathrm{N}$ e $\mathrm{K}_{2} \mathrm{O}$ foram realizadas ao longo do crescimento da cultura via fertirrigação. Nas parcelas em condições de sequeiro, a ureia e o cloreto de potássio foram aplicados em uma única vez, e para os tratamentos irrigados a aplicação foi feita fracionadamente após o corte da soqueira (DAC) para sistemas de plantio irrigado e sequeiro. As duas aplicações de $\mathrm{N} \mathrm{e} \mathrm{K}_{2} \mathrm{O}$ foram realizadas abrindo-se pequenos sulcos laterais à linha de plantio com enxada, após este procedimento os sulcos laterais foram rapidamente fechados evitandose assim possíveis perdas de $\mathrm{N}$ por volatilização.

O sistema de irrigação constituiu em tubos gotejadores enterrados a $25 \mathrm{~cm}$ de profundidade da superfície do solo, no meio da linha dupla. As características técnicas do tubo gotejador utilizado foram: tubo gotejador DripNet de parede delgada com $16 \mathrm{~mm}$ de diâmetro; vazão de $1 \mathrm{~L} \mathrm{~h}^{-1}$; pressão de serviço (PS) 31,6 m.c.a.; autocompensante e espaçamento entre gotejadores de $0,50 \mathrm{~m}$. As linhas laterais tiveram $30 \mathrm{~m}$ de comprimento, mantendo o espaçamento entre gotejadores original, com o intuito de não modificar as reais condições de fabricação. Para o manejo da irrigação utilizou-se o cálculo da evapotranspiração segundo Penman-Monteith (FAO), multiplicando pelo coeficiente de cultivo da cultura (Kc), próxima de 100\% da evapotranspiração da cultura por ciclo de crescimento (Gava et al., 2011; e Dellabiglia, 2016). Os dados climatológicos foram obtidos da estação meteorológica completa da FATEC/JAU localizada a 10 km da área experimental.

Os valores dos Kc para os estádios de desenvolvimento da cultura utilizados para os cálculos da Evapotranspiração da cultura (ETc) estão na Figuras 2 e 3 para o ambiente irrigado e sequeiro, respectivamente. 
Figura 2. Balanço hidrico durante a realiazação do experimento out15/nov16 para o sistema irrigado. Onde, Etc = evapotranspiração da cultura; $\mathrm{P}$ = precipitação; $\mathrm{I}$ = irrigação; $\mathrm{DEF}=$ deficiência hidrica.

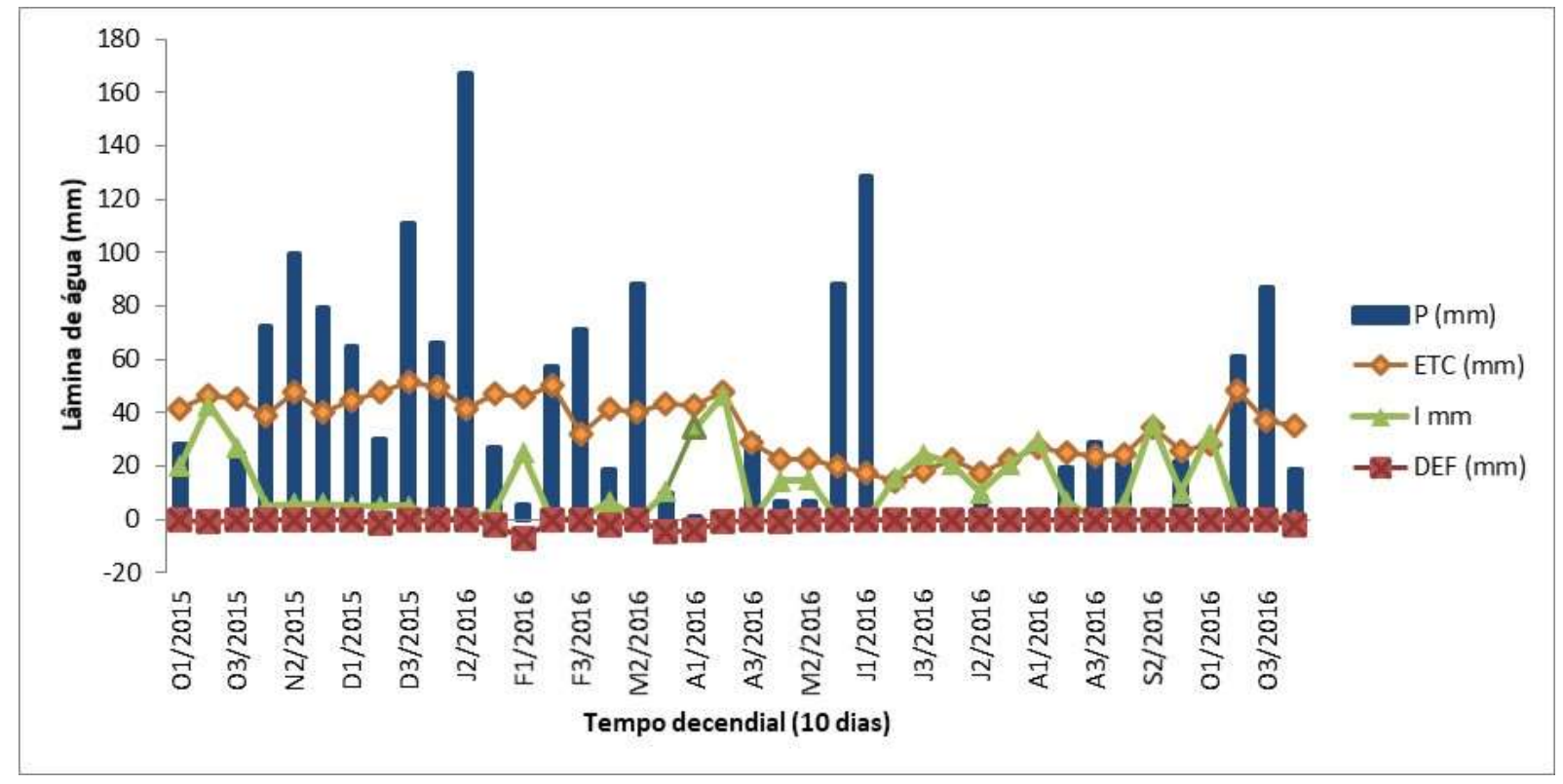

Fonte: Autores.

Figura 3. Balanço hídrico durante a realização do experimento out15/nov16 para o sistema sem irrigação (sequeiro). Onde, Etc = evapotranspiração da cultura; $\mathrm{P}=$ precipitação; $\mathrm{DEF}=$ deficiência hidrica.

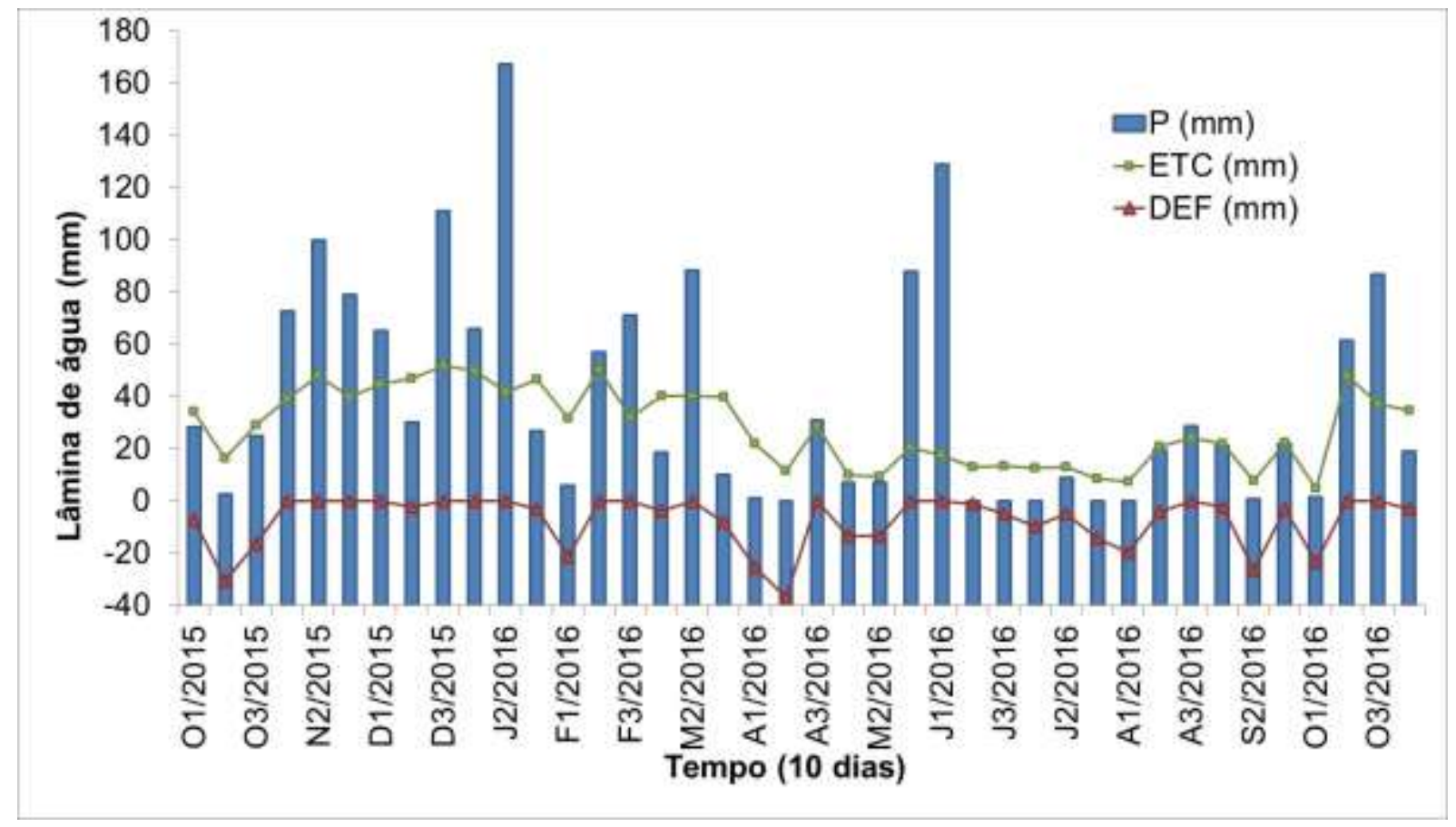

Fonte: Autores.

A frequência de irrigação foi realizada contabilizando-se o suprimento de água no solo, pela precipitação (P), e a demanda atmosférica pela evapotranspiração da cana-de-açúcar (ETC) que para o ambiente irrigado teve ETC igual a 1401,8 mm e para o ambiente não irrigado teve ETC igual a 1127,9 mm; considerando uma capacidade de água disponível do solo (CAD) de $70 \mathrm{~mm}$. Desse modo foi estimado o balanço hídrico a cada decêndio e calculada a deficiência hídrica (DEF), tanto 
para o tratamento irrigado com 26,5 mm (Figura 2), quanto para o tratamento não irrigado com 300,4 mm (Figura 3), através da diferença entre a evapotranspiração potencial (ETP) e a evapotranspiração real (ETR). A deficiência hídrica calculada para o período foi de $326,9 \mathrm{~mm}$.

As avaliações fisiológicas foram realizadas na cana-soca safra 2015/2016 e em diferentes fases fenológicas, dos 115 aos 393 dias após o corte, constituindo-se de quatro épocas de avaliações $\left(A_{1}, A_{2}, A_{3}\right.$ e $\left.A_{4}\right)$ durante o ciclo. Na colheita final $\left(\mathrm{A}_{4}\right)$ foram realizadas as avaliações qualitativas e quantitativas, aos 393 dias após o corte, sendo:

- Índice SPAD: determinada usando um clorofilômetro (modelo SPAD- 502, Konica Minolta, New Jersey, EUA). A leitura da parcela foi obtida por meio da média de 15 leituras realizadas na folha +3 (terceira folha apical completamente expandida), assumindo diferentes perfilhos em cada época de avaliação, aos 115, 235, 347 e 393 DAP.

- A condutância estomática (gs): foi obtida através de aparelho porômetro (Leaf Porometer - Decagon Devices). As leituras foram tomadas na região mediana da folha +1 e realizadas pela manhã antes das 10:00h, assumindo diferentes perfilhos em cada época de avaliação, aos 115, 235, 340 e 393 DAC. Os valores de temperatura foliar foram obtidos através de aparelho porômetro (Leaf Porometer - Decagon Devices). Os valores de temperatura foram obtidos simultaneamente às leituras de condutância estomática, utilizando-se a mesma região mediana da folha +1 e realizadas pela manhã antes das 10:00h, assumindo diferentes perfilhos em cada época de avaliação, aos 115, 235, 340 e 393 DAC.

- O índice de área foliar (IAF): determinado com o uso de um Ceptômetro. As leituras em cada parcela foram tomadas às 14:00h abaixo do dossel foliar nas épocas de avaliação após a implantação dos tratamentos. O IAF foi determinado por meio da diferença entre leituras de PAR acima e abaixo do dossel foliar.

Os resultados foram submetidos à análise de variância (ANOVA), ao nível de 5\% de significância, e as médias dos tratamentos qualitativos (irrigado ou sequeiro) foram comparadas através do teste Tukey $(p \leq 0,05)$. Para os dados referentes às doses de potássio, utilizou-se a análise de regressão polinomial linear e quadrática.

\section{Resultados e Discussão}

Para o índice SPAD não houve interação entre os fatores (ambiente: irrigado e sequeiro $x$ doses de $\mathrm{K}_{2} \mathrm{O}$ ). Houve efeito significativo somente para o fator ambiente (irrigado e sequeiro) aos $235 \mathrm{DAC}$, onde o tratamento irrigado foi superior ao de sequeiro em 9\% (Figura 4).

Figura 4. Índice SPAD medido aos 115, 235, 340 e 393 dias após a colheita em plantas de cana-de-açúcar cultivadas em ambiente irrigado e de sequeiro (sem irrigação).

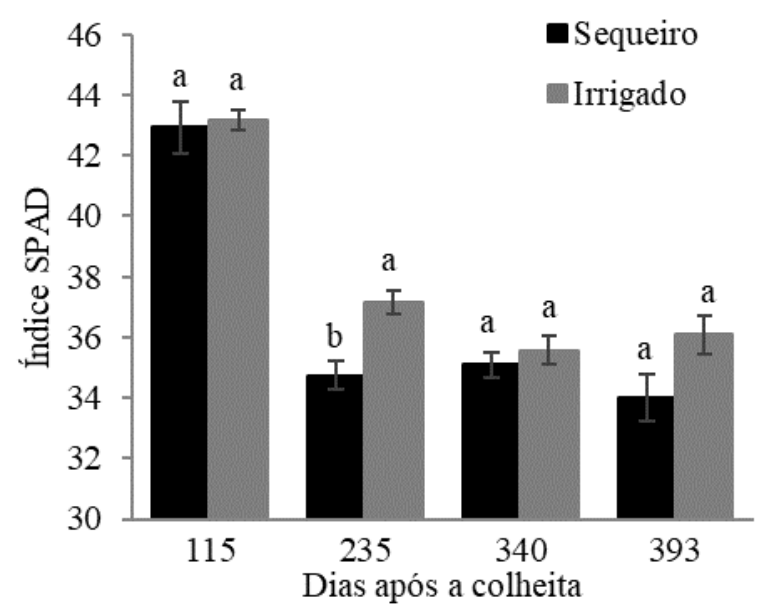

As letras diferentes evidenciam diferenças entre as médias ao nível de 10\% de significância. Fonte: Autores. 
Quando as leituras SPAD são inferiores a 40, indicam o início da deficiência de clorofila, o que afeta o processo fotossintético (Torres Netto et al., 2005). Apesar de não haver influência das doses de K nesta variável, podemos observar o índice SPAD ficou abaixo do esperado a partir dos 235 DAC, evidenciando um possível estresse hídrico a partir de 235 DAC, mesmo quando as plantas foram irrigadas, o que não esperado, pois os dados apresentados nas Figuras 2 e 3 contrapõem essa afirmativa. A redução da clorofila é um dos agravantes na cultura em virtude da deficiência hídrica, que resulta no baixo teor da cor verde nas folhas (Long et al., 1994). O uso dessa técnica também é de fácil obtenção e vem se mostrando confiável para diferenciar entre plantas tolerantes e susceptíveis à deficiência hídrica (O’Neill et al., 2006).

Para a condutância estomática (gs) houve efeito significativo para ambiente (irrigado e sequeiro) em todas as épocas de avaliação, porém, só houve efeito significativo isolado do $\mathrm{K}_{2} \mathrm{O}$ nas avaliações de 115,340 e 393 DAC. A interação só foi verificada aos 393 DAC. Aos 340 DAC, tanto no tratamento irrigado quanto no sequeiro, maiores doses acarretaram maior condutância (Tabela 2). Nos demais períodos que apresentaram diferenças (115 e 235), eles ocorreram somente no tratamento irrigado.

Tabela 2. Médias de condutância estomática medida aos 115, 235, 340 e 393 dias após a colheita (DAC) em plantas de canade-açúcar cultivadas em diferentes ambientes (irrigado e "sequeiro - sem irrigação") em diferentes doses de potássio.

\begin{tabular}{|c|c|c|c|c|c|}
\hline \multirow{3}{*}{ DAC } & \multirow{3}{*}{ Ambiente } & \multicolumn{4}{|c|}{ Doses de potássio $\left(\mathrm{Kg} \mathrm{ha}^{-1}\right)$} \\
\hline & & 0 & 70 & 140 & 210 \\
\hline & & \multicolumn{4}{|c|}{ Médias } \\
\hline \multirow{2}{*}{115} & Sequeiro & $164,18 b$ & $171,20 \mathrm{~b}$ & $194,53 \mathrm{~b}$ & $216,78 \mathrm{~b}$ \\
\hline & Irrigado & 249,88 a & $281,88 \mathrm{a}$ & 297,33 a & $364,63 \mathrm{a}$ \\
\hline \multirow{2}{*}{235} & Sequeiro & $97,38 \mathrm{a}$ & $114,80 \mathrm{~b}$ & $110,60 \mathrm{~b}$ & $107,50 \mathrm{~b}$ \\
\hline & Irrigado & $123,78 \mathrm{a}$ & 156,48 a & $160,48 \mathrm{a}$ & $169,05 \mathrm{a}$ \\
\hline \multirow{2}{*}{340} & Sequeiro & $77,85 \mathrm{~b}$ & $113,03 \mathrm{~b}$ & $165,23 \mathrm{~b}$ & $160,20 \mathrm{~b}$ \\
\hline & Irrigado & $136,80 \mathrm{a}$ & $187,95 \mathrm{a}$ & 227,08 a & $224,93 \mathrm{a}$ \\
\hline \multirow{2}{*}{393} & Sequeiro & $107,23 \mathrm{a}$ & $171,48 \mathrm{~b}$ & $132,68 \mathrm{~b}$ & $130,55 \mathrm{~b}$ \\
\hline & Irrigado & $144,55 \mathrm{a}$ & $259,78 \mathrm{a}$ & $320,88 \mathrm{a}$ & $240,08 \mathrm{a}$ \\
\hline
\end{tabular}

As letras diferentes na mesma coluna representam diferença significativa pelo teste Tukey $(p \leq 0,01)$ entre ambientes em função das doses de potássio.Fonte: Autores.

A maior dose de adubação potássica $\left(210 \mathrm{~kg} \mathrm{ha}^{-1}\right)$ proporcionou um aumento na condutância estomática quando comparado a 0 de aplicação no ambiente de sequeiro, quase duplicando o valor da gs, denotando o efeito positivo do $\mathrm{K}^{+}$às plantas que estão sofrendo um estresse hídrico. Assim como este efeito também pode ser visualizado num ambiente irrigado, porém, não de forma tão acentuada. Segundo Prado (2003), as plantas bem supridas de potássio apresentam maior eficiência no uso da água, enquanto plantas que estão sob deficiência de potássio possuem menor desempenho fotossintético, devido à abertura estomática não acontecer de forma regular, reduzindo a entrada de $\mathrm{CO}_{2}$.

Isso mostra que a adubação potássica melhora mais a gs na cultura de sequeiro do que irrigada, porém os valores da gs quando irrigado foram sempre superiores ao não irrigado, como já supracitado. 
A superioridade da gs no tratamento irrigado em comparação ao sequeiro foi de 59,5\% em média $\left(111,8 \mathrm{mmol} \mathrm{m}^{-2} \mathrm{~s}^{-1}\right)$ para $115 \mathrm{DAC}, 41,4 \%\left(44,8 \mathrm{mmol} \mathrm{m}^{-2} \mathrm{~s}^{-1}\right)$ para $235 \mathrm{DAC}, 55,0 \%\left(65,1 \mathrm{mmol} \mathrm{m}^{-2} \mathrm{~s}^{-1}\right)$ para $340 \mathrm{DAC}$ e $78,0 \%\left(105,8 \mathrm{mmol} \mathrm{m}^{-2} \mathrm{~s}^{-}\right.$ ${ }^{1}$ ) para 393 DAC (Figura 5). Fatos que podem ser justificados pelo decréscimo na disponibilidade de água no solo que ocasiona a redução no potencial da água nas folhas das plantas, levando à perda de turgescência e à redução da condutância estomática (Shalhevet, 1983). A redução da taxa de assimilação de $\mathrm{CO}_{2}$ durante o estresse hídrico se deve à redução na disponibilidade de $\mathrm{CO}_{2}$ no interior da folha, causada pelo fechamento dos estômatos em resposta à redução da disponibilidade de água no solo (Rosa et al., 1991). Esse aumento da resistência à difusão gasosa e a diminuição na taxa de assimilação de $\mathrm{CO}_{2}$ determina menor perda de água por transpiração, além de poder afetar a fotossíntese.

Figura 5. Condutância estomática medida aos 115, 235, 340 e 393 dias após a colheita em plantas de cana-de-açúcar cultivadas em ambiente irrigado e de sequeiro (sem irrigação).

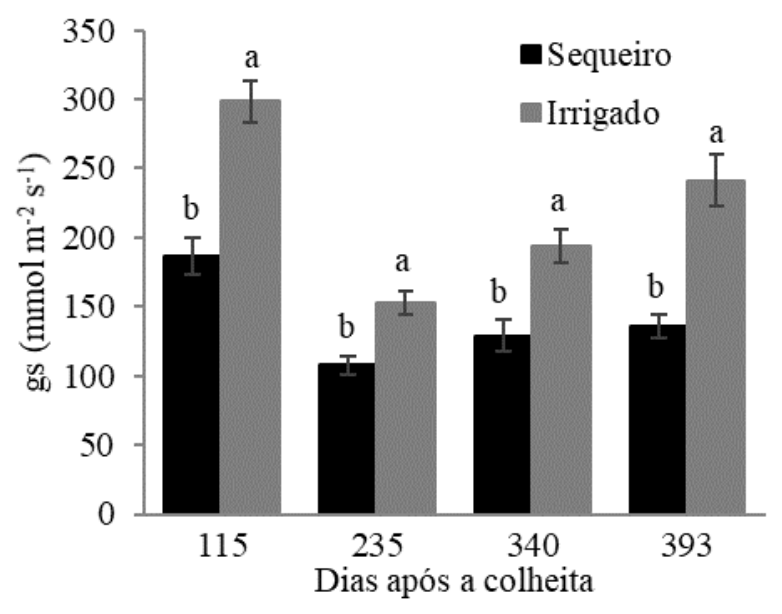

As letras diferentes representam diferença significativa entre valores médios ao nível de $10 \%$ de significância pelo teste de Tukey. Fonte: Autores.

Para a gs medida aos 115 e 340 DAC houve um aumento linear com o aumento das doses de potássio (Figura 6). Isso mostra que doses de até $210 \mathrm{~kg} \mathrm{ha}^{-1}$ aumentou a resistência da cana-de-açúcar ao estresse hídrico.

Figura 6. Condutância estomática (gs) medida aos 115 e 340 dias após a colheita da cana-de-açúcar (A e B, respectivamente) em função de diferentes doses de potássio.
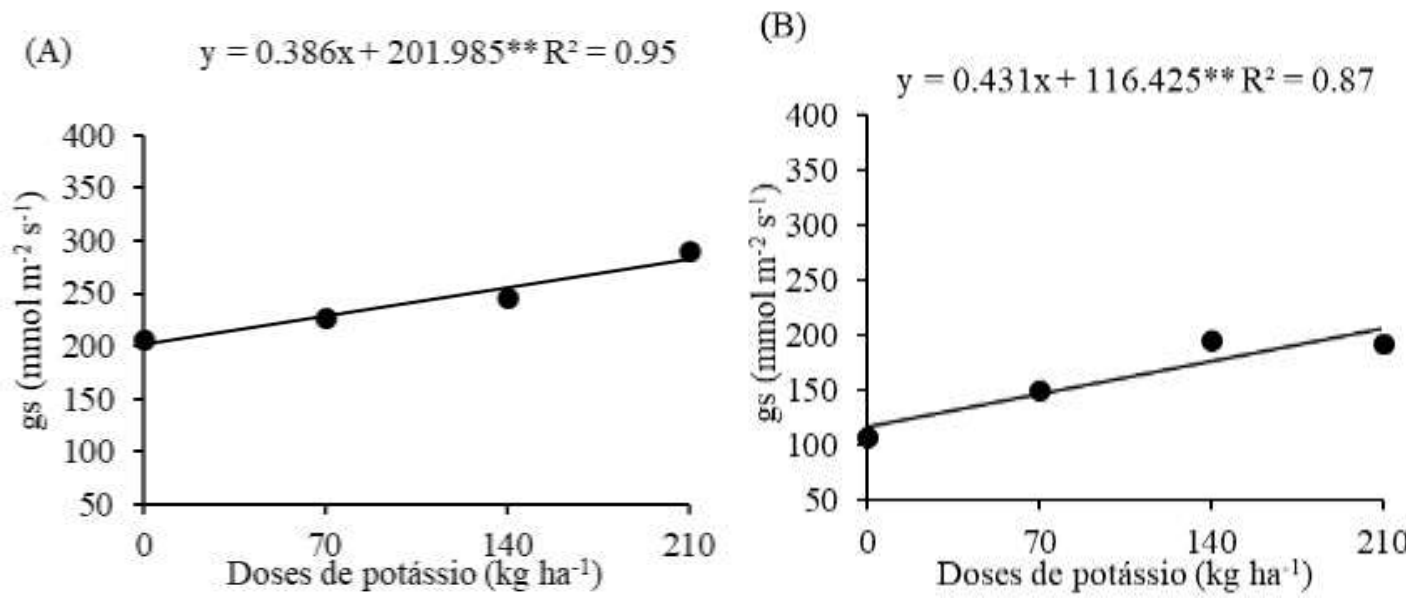

**, * e $\uparrow$ : significativo ao nível de significância de 1, 5 e 10\% respectivamente. Fonte: Autores. 
Aos 393 DAC houve um ajuste quadrático (Figura 7) para a cultura em sequeiro e irrigada, onde as doses de potássio proporcionaram a maior gs em, aproximadamente, 100 e $130 \mathrm{~kg} \mathrm{ha}^{-1}$ de $\mathrm{K}_{2} \mathrm{O}$, respectivamente.

Figura 7. Condutância estomática (gs) medida aos 393 dias após a colheita da cana-de-açúcar em função de diferentes doses de potássio em ambiente de sequeiro (S) e irrigado (I)].

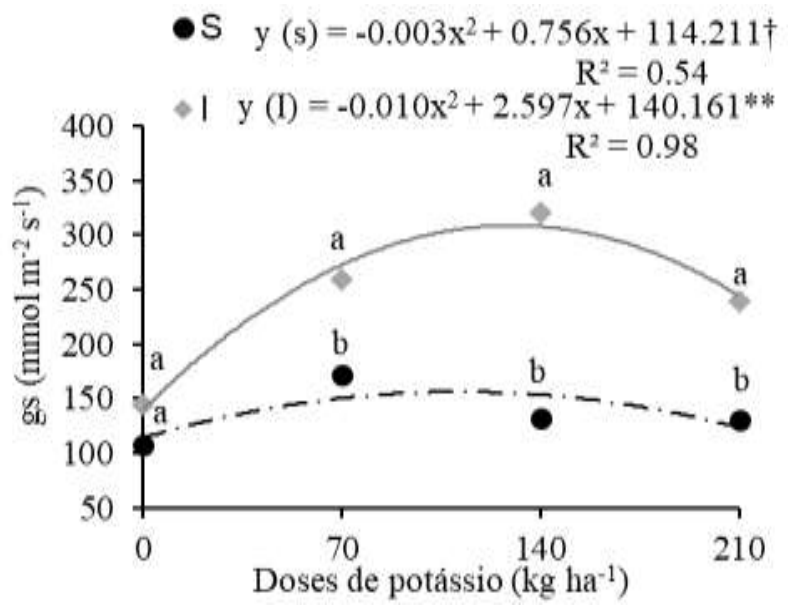

As letras diferentes em uma mesma dose de potássio representam diferença significativa entre valores médios ao nível de até $10 \%$ de significância. ${ }^{* *}, *$ e †: significativo ao nível de significância de $1 ; 5$ e $10 \%$ respectivamente.Fonte: Autores.

Na Figura 7, percebe-se que o ambiente irrigado foi superior ao sequeiro, considerando a disponibilidade hídrica da área em estudo, visto que para elevadas doses de $\mathrm{K}_{2} \mathrm{O}$ foram onde obteve-se a maior significância, o mesmo comportamento observado por (Zanchim, 2015). Resultados similares também foram encontrados por outros pesquisadores, os quais constataram a redução da condutância estomática em cana-de-açúcar sob deficiência hídrica (Pincelli \& Silva, 2012; Brunelli, 2014; Geronimo, 2014), bem como a redução da condutância estomática ao longo do tempo. Pois o fechamento dos estômatos é um mecanismo de defesa da planta, e tem por objetivo reduzir a perda de água, porém como consequência reduz também a assimilação de $\mathrm{CO}_{2}$ (Pimentel, 2004).

Em relação a temperatura foliar (Tf) houve efeito significativo para o ambiente aos 235, 340 e 393 DAC e para as doses apenas aos 115 DAC. Houve diferenças entre sequeiro e irrigado para as doses de potássio. A superioridade da Tf no tratamento sequeiro frente ao irrigado foram de 8,1\% (1,9) para 235 DAC, 7,5\% $(2,5)$ para 340 DAC e 10,3\% $(3,4)$ para 393 DAC (Figura 8A). Esses resultados corroboram com Trentin et al. (2011), em estudo realizado em casa de vegetação com cana-de-açúcar, cultivar RB 86-7515, em que as plantas mantidas sob adequado fornecimento hídrico apresentaram temperaturas inferiores, em torno de $6,6^{\circ} \mathrm{C}$, quando comparadas àquelas sob condições de estresse hídrico severo e elevada radiação solar. 
Figura 8. Temperatura foliar medida aos 115, 235, 340 e 393 dias após a colheita em plantas de cana-de-açúcar cultivadas em ambiente irrigado e de sequeiro (a esquerda) e medida aos 115 dias após a colheita em função de doses de potássio (a direita).
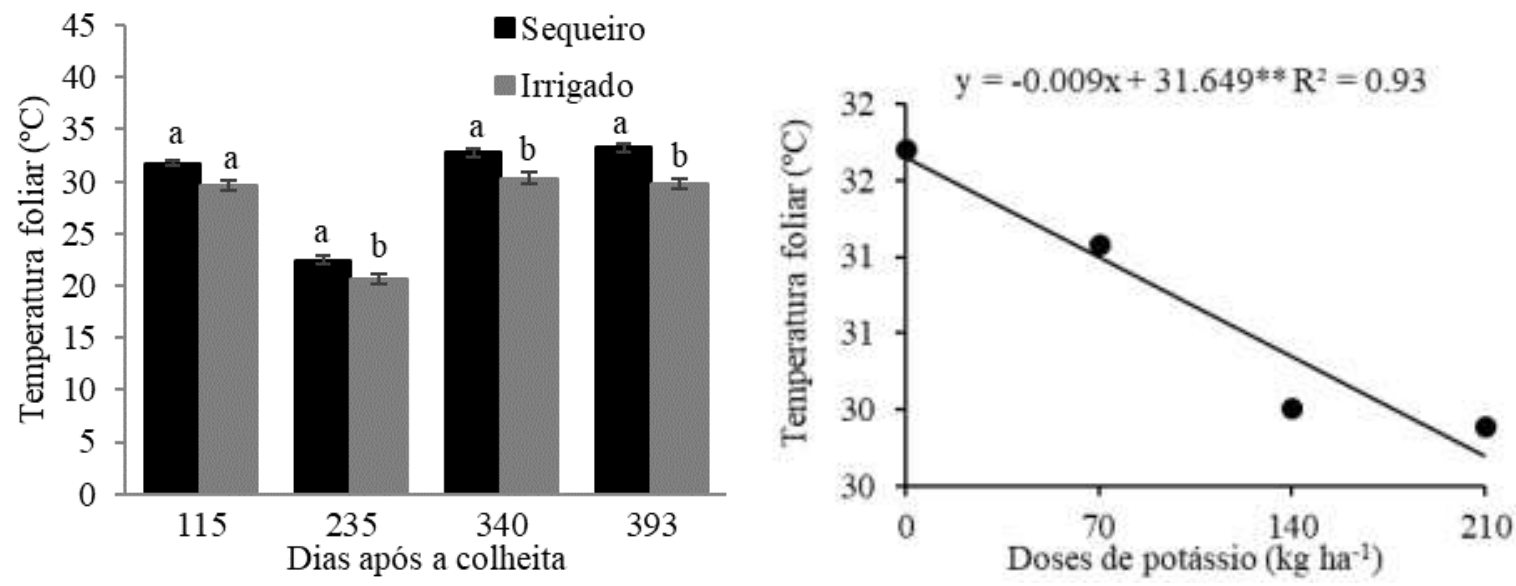

Letras diferentes evidenciam diferenças entre valores médios ao nível de $10 \%$ de significância. **, * e †: significativo ao nível de significância de 1, 5 e 10\% respectivamente; ns: não significativo. Fonte: Autores.

As doses de potássio somente surtiram efeito significativo na temperatura foliar no tratamento sequeiro aos $115 \mathrm{DAC}$, com um decréscimo linear da Tf com o aumento nas doses de potássio (Figura 8B).

Para o índice de área foliar (IAF) houve efeito significativo dos ambientes estudados apenas aos 393 DAC e para a dose de $140 \mathrm{~kg} \mathrm{ha}^{-1}$ de $\mathrm{K}$ aos $115 \mathrm{DAC}$, sendo que a superioridade do tratamento irrigado frente ao sequeiro, neste caso, foi de 9\% $\left(3 \mathrm{~m}^{2} \mathrm{~m}^{-2}\right.$ ) (Figura 9A). Alguns fatores como temperaturas elevadas em períodos de estresse hídrico causam a diminuição da área foliar, pois aceleram o processo de senescência das folhas verdes (Inmam-Bamber 2004). Analisando a Tf, pode-se notar que esta foi a data que houve maior discrepância de temperatura entre os ambientes.

Na Figura 9A pode-se perceber a diferença entres os ambientes irrigado e sequeiro, onde a superioridade da variável no tratamento irrigado frente ao sequeiro foi de $18,2 \%(1,1)$ para 393 DAC.

Figura 9. Índice de área foliar (IAF) medido aos 115, 235, 340 e 393 dias após a colheita em plantas de cana-de-açúcar cultivadas em ambiente irrigado e de sequeiro (a esquerda) e medido aos 115 dias após a colheita em função de doses de potássio (a direita).
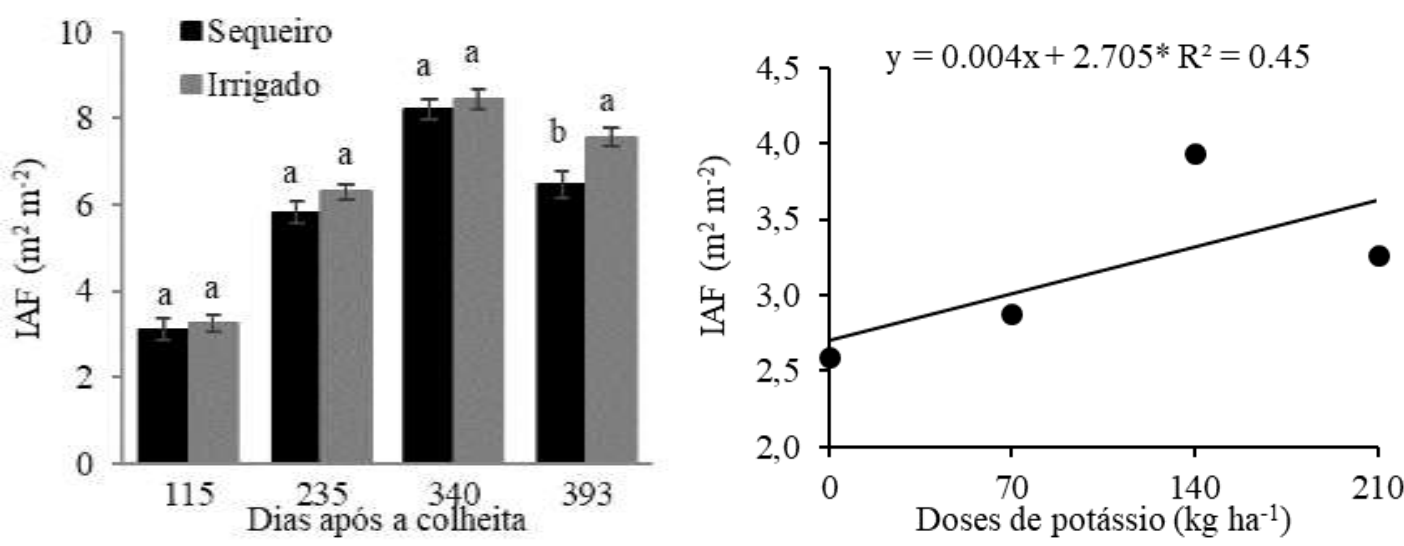

As letras diferentes evidenciam diferenças entre valores médios ao nível de $10 \%$ de significância. **, * e $\dagger$ : significativo ao nível de significância de 1, 5 e $10 \%$ respectivamente. Fonte: Autores. 
Um aumento linear do IAF foi verificado com o aumento das doses de potássio ( $p<0,05)$ aos 115 DAC (Figura 9 B), demonstrando um possível efeito benéfico da aplicação de $\mathrm{K}_{2} \mathrm{O}$ no solo. A área foliar está diretamente relacionada com o comprimento foliar, e em plantas com deficiência de $\mathrm{K}$, a redução desses parâmetros deve-se, principalmente, pela redução da taxa de alongamento foliar, o que estaria mais diretamente relacionado com a alteração da relação de água na planta, do que pela redução na taxa de suprimentos de carboidratos (Gerardeaux et al., 2010).

\section{Conclusão}

A condutância estomática, temperatura foliar e o índice de área foliar da cana-de-açúcar são diferentes na maioria das épocas amostradas a partir dos 235 dias após o corte.

O índice SPAD nas plantas de cana-de-açúcar não foi influenciado pelas doses de potássio nos dois regimes hídricos.

As doses de potássio não alteraram a qualidade tecnológica da cana-de-açúcar. As doses de 70 e $210 \mathrm{~kg} \mathrm{ha}^{-1} \mathrm{de}$ potássio produziram maiores quantidades de colmos e de açúcar, respectivamente, para ambientes cultivados em sequeiro e irrigado.

\section{Agradecimentos}

Os autores agradecem à Fundação de Amparo à Pesquisa do Brasil (Conselho Nacional de Desenvolvimento Científico e Tecnológico - CNPq), à Coordenação de Aperfeiçoamento de Pessoal de Nível Superior (CAPES); a Fundação de Amparo à Pesquisa do Estado de Goiás (FAPEG); a Coordenação de Aperfeiçoamento de Pessoal de Nível Superior (Capes); a Financiadora de Estudos e Projetos (Finep); Centro de Excelência em Agro Exponencial (CEAGRE) e ao Instituto Federal Goiano pelo apoio financeiro e logístico.

\section{Referências}

Brunelli, M. C. (2014). Índice spad e trocas gasosas ao longo do dia para diferenciação de cana-de-açúcar sob deficiência hídrica. 2014. 63 f. Dissertação (Mestrado em Agronomia- Agricultura) - Faculdade de Ciências Agronômicas, Universidade Estadual Paulista, Botucatu.

Cattivelli, L., Rizza, F., Badeck, F. W., Mazzucotelli, E., Mastrangelo, A. M., Francia, E., \& Mare, C. (2008). Drought tolerance improvement in crop plants: An integrated view from breeding to genomics. Field Crops Research, Amsterdam, v.105(1-2), 1-14.

Có Júnior, C., Marques, M. O., \& Tasso Júnior, L. C. (2008). Efeito residual de quatro aplicações anuais de lodo de esgoto e vinhaça na qualidade tecnológica da cana-de-açúcar. Revista Engenharia Agrícola, Jaboticabal, v. 28(1), 196-203.

CONAB (2017). Acompanhamento da safra brasileira de cana-de-açúcar. Safra 2015/16. Quarto Levantamento, 3(4). Brasília, abril/2017. http://www.udop.com.br/download/estatistica/conab_levantamento_safras/17abr17_4o_levantamento_safra_2016a2017.pdf.

Dalri, A. B., \& Cruz, R. L. (2008). Produtividade da cana-de-açúcar fertirrigada com N e K via gotejamento subsuperficial. Revista Engenharia Agrícola, Jaboticabal, v. 28(3), 516-524.

Dellabiglia, W. J. (2016). Disponibilidade hídrica e utilização do nitrogênio em cana-de-açúcar irrigada por gotejamento subsuperficial. 2016. 79f. Dissertação (Mestrado em Agronomia). Universidade Estadual Paulista. Botucatu, São Paulo.

Gava, G. J. C., Silva, M. A., Silva, R. C., Jeronimo, E. M., Cruz, J. C. S., \& Kölln, O. T. (2011). Produtividade de três cultivares de cana-de-açúcar sob manejos de sequeiro e irrigado por gotejamento. Revista Brasileira de Engenharia Agrícola e Ambiental, Campina Grande, v. 15(3), $250-255$.

Geronimo, G. Z. (2014). Características morfofisiológicas e agrupamento genético de cana-de-açúcar sob deficiência hídrica. 2014. 81f. Dissertação (Mestrado em Agronomia - Agricultura) - Faculdade de Ciências Agronômicas, Universidade Estadual Paulista, Botucatu.

Howell, T. A., \& Evett S. R. (2004). The Penman-Monteith method. Washington, DC: USDA-Agricultural Research Service, Conservation \& Production Research Laboratory, 14.

Lima, C. C. (2012). Características Fenológicas de Cana de Açúcar Adubada com Fertilizantes Minerais Adicionados de Substâncias Húmicas. - FERTBIO 2012 Maceió (AL), 17 a 21 de setembro de 2012.

Malavolta, E., \& Crocomo, O. J. (1982). Funções do potássio na planta. In: Yamada, T.; Igue, K.; Muzilli, O.; Usherwood, N. R. (Eds.). Potássio na Agricultura Brasileira. Piracicaba: Instituto da Potassa e Fosfato (EUA), 95-162. 
Research, Society and Development, v. 10, n. 14, e549101422526, 2021

(CC BY 4.0) | ISSN 2525-3409 | DOI: http://dx.doi.org/10.33448/rsd-v10i14.22526

Oliveira, E. C. A. (2011). Balanço nutricional da cana-de-açúcar relacionada à adubação nitrogenada. 2011. 213 f. Tese (Doutorado em Solos e Nutrição de Plantas) - Escola Superior de Agricultura "Luiz de Queiroz", Universidade de São Paulo, Piracicaba.

Oddo, E., Inzerillo S., Grisafi F., Sajeva M., Salleo S., \& Nardini A. (2014). Does short-term potassium fertilization improve recovery from drought stress in laurel. Tree Physiology, Oxford, v. 34(8), 906-913.

O'neill, P. M., Shanahan, J. F., \& Schepers, J. S. (2006). Use of chlorophyll fluorescence assessments to differentiate corn hybrid response to variable water conditions. Crop Science, Madison, v. 46(1), 681-687.

Pimentel, C. (2004). A relação da planta com a água. (1ª ed.) Seropédica: EDUR, 191.

Pincelli, R. P., \& Silva, M. A. (2012). Alterações morfológicas foliares em cultivares de cana-de-açúcar em resposta à deficiência hídrica. Bioscience Journal, Uberlândia, v. 28(4), 546-556.

Prado, H. (2003). Ambientes de produção de cana-de-açúcar. Bebedouro. http://www.revistacoopercitrus.com.br//edicao202/Ambien_m_11.asp.

Raij, B. V., Andrade, J. C., Cantarella, H., \& Quaggio, J. A. (Ed.) (2001). Análise química para avaliação da fertilidade de solos tropicais. Campinas: IAC, 285 .

Ridesa (2010). Rede Interuniversitária para o Desenvolvimento do Setor Sucroalcooleiro. Catálogo nacional de variedades "RB" de cana-de-açúcar. Curitiba: RIDESA, 136.

Rosa, L. M., Dillenburg, L. R., \& Forseth, I. N. (1991). Responses of soybean leaf angle, photosynthesis and stomatal conductance to leaf and soil water potential. Annals of Botany, London, v. 67(1), 51-8.

Shabala, S., \& Pottosin, I. (2014). Regulation of potassium transport in plants under hostile conditions: Implications for abiotic and biotic stress tolerance. Physiologia Plantarum, Copenhagen, v. 151(3), 257-279.

Shalhevet, J. (1983). Plants under salt and water stress. In: Fowden, L.; Mansfield, T., \& Stoddart, J. Plant adaptation to environmental stress. London: Chapman \& Hall, 133-54.

Silva, M. A., Arantes, M. T., Rhein, A. F. L.,Gava, G. J. C., \& Kolln, O. T. (2014). Potencial produtivo da cana-de-açúcar sob irrigação por gotejamento em função de variedades e ciclos. Revista Brasileira de Engenharia Agrícola e Ambiental, Campina Grande, v.18(3), 241-249.

Silva, M. A., Jifon, J. L., Silva, J. A. G., \& Santos, C. M. (2014). Relationships between physiological traits and productivity of sugarcane in response to water deficit. The Journal of Agricultural Science, Cambridge, v. 152(1), 104-118.

Taulya, G. (2013). East African highland bananas (Musa spp. AAA-EA) ‘worry’ more about potassium deficiency than drought stress. Field Crops Research, Amsterdam, v. 151, 45-55.

Torres Netto, A., Campostrini, E., Oliveira, J. G., \& Bressan-Smith, R. E. (2005). Photosynthetic pigments, nitrogen, chlorophyll a fluorescence and SPAD502 readings in coffee leaves. Scientia Horticulturae, Amsterdam, v. 104(2), 199-209.

Zanchim, B. J. (2015). Eficiência de uso de potássio e de água em genótipos de cana-de-açúcar. 2015. 115f. Dissertação (Mestrado em Ciências/Biologia na Agricultura e Ambiente). Centro de Energia Nuclear na Agricultura, Universidade de São Paulo, Piracicaba. 